\title{
Peristaltic Elastic Instability in an Inflated Cylindrical Channel
}

\author{
Nontawit Cheewaruangroj \\ Cavendish Laboratory, University of Cambridge, $19 \mathrm{JJ}$ Thomson Avenue, Cambridge CB3 OHE, United Kingdom \\ Karolis Leonavicius and Shankar Srinivas \\ Department of Physiology Anatomy and Genetics, University of Oxford, Oxford OX1 3QX, United Kingdom \\ John S. Biggins \\ Department of Engineering, University of Cambridge, Trumpington Street, Cambridge CB2 1PZ, United Kingdom
}

(Received 8 May 2018; revised manuscript received 21 November 2018; published 13 February 2019)

\begin{abstract}
A long cylindrical cavity through a soft solid forms a soft microfluidic channel, or models a vascular capillary. We observe experimentally that, when such a channel bears a pressurized fluid, it first dilates homogeneously, but then becomes unstable to a peristaltic elastic instability. We combine theory and numerics to fully characterize the instability in a channel with initial radius $a$ through an incompressible bulk neo-Hookean solid with shear modulus $\mu$. We show instability occurs supercritically with wavelength $12.278 \ldots a$ when the cavity pressure exceeds $2.052 \ldots \mu$. In finite solids, the wavelength for peristalsis lengthens, with peristalsis ultimately being replaced by a long-wavelength bulging instability in thin-walled cylinders. Peristalsis persists in Gent strain-stiffening materials, provided the material can sustain extension by more than a factor of 6 . Although naively a pressure driven failure mode of soft channels, the instability also offers a route to fabricate periodically undulating channels, producing, e.g., waveguides with photonic or phononic stop bands.
\end{abstract}

DOI: 10.1103/PhysRevLett.122.068003

A channel through a soft solid is the prototypical element of biological plumbing [1], guiding fluid through the vascular, lymphatic, digestive, reproductive, renal, and respiratory systems. Soft channels also underpin the blossoming field of soft microfluidics [2], which exploits the convenience of soft lithography for rapid prototyping [3,4], the deformability of soft channels to actuate valves and pumps $[2,5,6]$, and the mechanical compatibility between soft solids and soft tissues to build organs on chips and medical implants $[7,8]$. Here we investigate how soft channels deform as their internal pressure increases. Thin-walled elastic tubes famously undergo long-wavelength bulging, bending, and ballooning instabilities [9-18] under inflation, but a channel through a bulk solid arises in the opposite (divergently thick-walled) limit. We combine theory, numerics, and experiment (Fig. 1) to show that, while modest fluid pressure causes simple dilation, the channel undergoes a finite-wavelength elastic instability and adopts a peristaltically undulating morphology when the pressure becomes comparable to the solid's shear modulus,

The hallmark of soft solids, including elastomers, gels, and many biological tissues, is that they can sustain geometrically large elastic strains ( $\gtrsim 100 \%$ ) without failing, exposing them to the full complexity of large deformation geometry. This generates a range of geometrically motivated elastic instabilities, including buckling [19], creasing [20-25], and wrinkling [26,27] under compression, fingering [28-32], fringing [33,34], and beading [35-38] under tension, and ballooning $[9,13,16,18,39]$, aneurysm [39,40], and cavitation [41-43] under inflation. These instabilities are important failure modes [44], but have also been exploited by evolution to sculpt developing brains $[45,46]$, guts $[47,48]$, and other

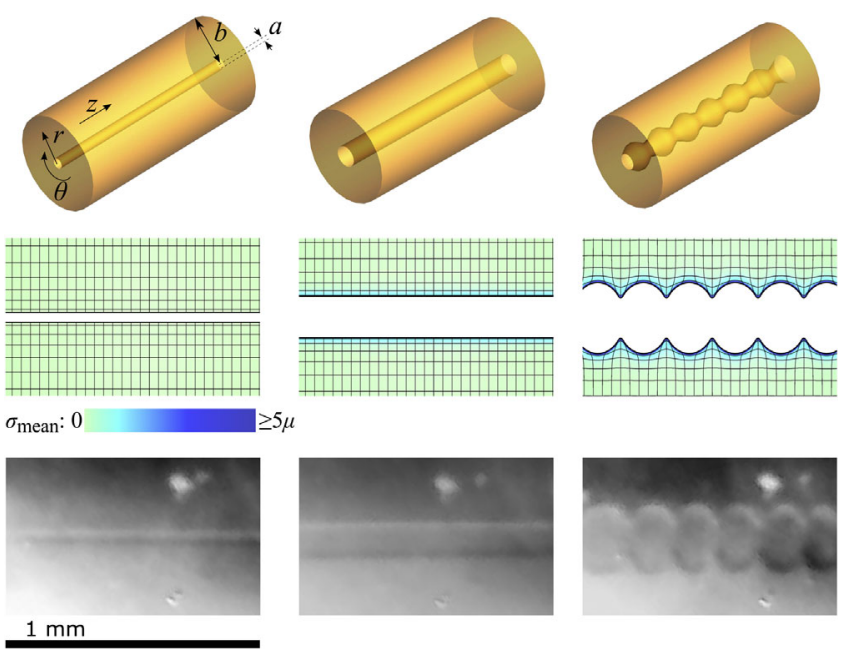

FIG. 1. Schematic (top), numerics (middle, neo-Hookean), and experiment (bottom, polyacrylamide) showing the shape evolution of a cylindrical channel through a soft solid under increasing internal pressure. At modest pressure the channel dilates simply, but at high pressures it undergoes an elastic instability and adopts a peristaltic shape (right). 
organs [49-53], and by engineers to make shape-switching devices [54-57].

We start by considering a cylindrical cavity, with initial radius $a$, running through soft elastic material occupying $a<r<b$, as shown in Fig. 1. If the channel is subject to an internal pressure $P_{\text {in }}$, it will dilate, deforming the solid and causing it to store elastic energy $E_{\mathrm{el}}$. The observed dilation will be determined by the minimum of the effective energy,

$$
E_{\mathrm{tot}}=E_{\mathrm{el}}-P_{\mathrm{in}} V,
$$

where $V$ is the dilated channel's volume. If the deformed solid has displacement $\mathbf{u}$, its local shape change is described by the deformation gradient tensor $F=I+\nabla \mathbf{u}$ ( $I$ being the identity matrix and $\nabla$ the gradient operator) and its elastic energy density is of the form $W(F)$. We model the solid as an incompressible $[\operatorname{Det}(F)=1]$ neo-Hookean material [58] with shear modulus $\mu$, requiring $W(F)=\frac{1}{2} \mu \operatorname{Tr}\left(F \cdot F^{T}\right)$ $P[\operatorname{Det}(F)-1]$, where $P$ is a Lagrange multiplier pressure field enforcing incompressibility. This model is exact for Gaussian polymer networks, and valid until deformations of several hundred percent in lightly cross-linked gels or elastomers [59], including our polyacrylamide gels [60]. It is also the simplest large-deformation elastic model, and correspondingly offers the clearest exposition of geometrically motivated instabilities.

Minimizing $E_{\text {tot }}$ with respect to variations in $\mathbf{u}$ and $P$ gives the expected equations of mechanical equilibrium, and the constraint of incompressibility,

$$
\nabla \cdot \sigma=0, \quad \operatorname{Det}(F)=1,
$$

where $\sigma=(\partial W / \partial F)=\left(\mu F-P F^{-T}\right)$ is the large deformation "first Piola-Kirchhoff" stress, a two-point tensor relating forces in the deformed configuration to reference configuration areas. These equations are augmented by the natural inner and outer boundary conditions:

$$
\left.\left(\sigma+P_{f} F^{-T}\right) \cdot \hat{\mathbf{r}}\right|_{r=a, b}=0,
$$

where the boundary fluid pressure $P_{f}=P_{\text {in }}, 0$ at $r=a, b$, respectively, and $\hat{\mathbf{r}}$ is the radial unit vector.

We first consider a simple radial dilation: $\mathbf{u}=u_{0}(r) \hat{\mathbf{r}}$, $P=\mu P_{0}(r)$. Incompressibility requires

$$
R \equiv r+u_{0}(r)=\sqrt{c^{2}+r^{2}}
$$

where the constant of integration $c$ parametrizes the degree of cavity dilation, with the inner radius rising by a factor of $\lambda=\sqrt{1+c^{2} / a^{2}}$. Mechanical equilibrium $(\nabla \cdot \sigma=0)$ then gives the form of the pressure field which, taking account of the stress-free outer boundary, is

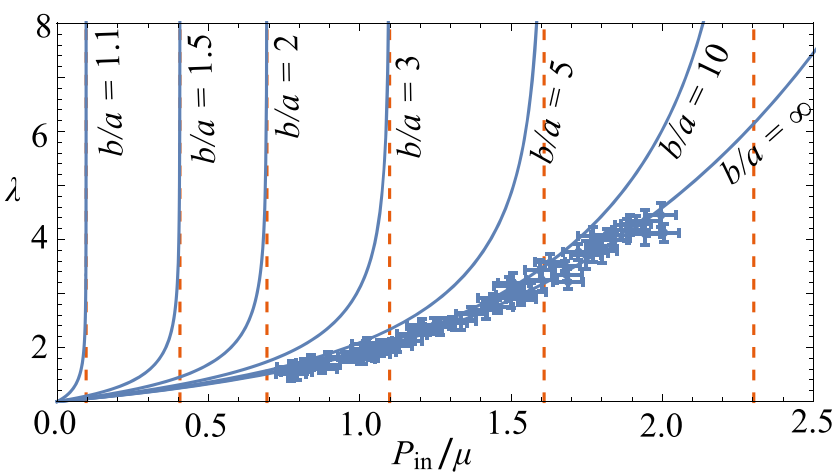

FIG. 2. Dilation factor $\lambda$ of a neo-Hookean cylindrical channel as a function of its interior pressure $P_{\text {in }}$, for a range of aspect ratios $b / a$. Predicted dilation (solid lines) diverges at $P_{\text {in }}=\mu \log (b / a)$, but a channel through a bulk solid $(b \rightarrow \infty)$ never diverges. Experimental points are individual constantpressure measurements from three channels through polyacrylamide slabs, reproducing the bulk neo-Hookean theoretical dilation.

$$
P_{0}=\frac{1}{2}\left[\frac{r^{2}}{c^{2}+r^{2}}+\frac{b^{2}}{c^{2}+b^{2}}+\ln \left(\frac{b^{2}\left(c^{2}+r^{2}\right)}{r^{2}\left(c^{2}+b^{2}\right)}\right)\right] .
$$

Finally, the inner boundary condition gives an implicit solution for the channel dilation $\lambda$, which, introducing $g[x] \equiv x^{-1}-\log (x)$, can be written as

$$
P_{\text {in }}=\frac{\mu}{2}\left\{g\left[1+(a / b)^{2}\left(\lambda^{2}-1\right)\right]-g\left[\lambda^{2}\right]\right\} .
$$

This predicted dilation is plotted for a range of values of $a / b$ in Fig. 2. Dilation rises monotonically with $P_{\text {in }}$, and diverges at $P_{\text {in }}=\mu \log (b / a)$. If $b=a+t \approx a$ (a pipe with thin wall thickness $t$ ), this critical pressure reduces to $P_{\text {in }} \approx \mu t / a$, the signature scaling of elastic instabilities in membrane tubes [9-18]. Conversely, in the $b \rightarrow \infty$ limit, Eq. (6) becomes

$$
P_{\text {in }}=\frac{\mu}{2}\left[1+\log \left(\lambda^{2}\right)-\lambda^{-2}\right],
$$

showing that the channel dilates, but only diverges at infinite pressure. In contrast, the result for a spherical cavity in a bulk solid, $P_{\text {in }}=(\mu / 2)\left[5-4 \lambda^{-1}-\lambda^{-4}\right]$, diverges at $P_{\text {in }}=\frac{5}{2} \mu$, a celebrated result known as solid cavitation [41].

We investigated the stability of cylindrical cavities under inflation experimentally by cross-linking a 1-mm-thick rectangular slab of polyacrylamide gel around a $30 \mu \mathrm{m}$ wire [further details in Supplemental Material [61]]. The wire was then removed (leaving a cylindrical channel), and the gel was equilibrated in a phosphate buffered saline solution (PBS). Finally, one end of the channel was plugged with a glass bead, and the channel was inflated by pumping additional PBS in through a glass capillary inserted at the other end. We used a computer-controlled air pressure 

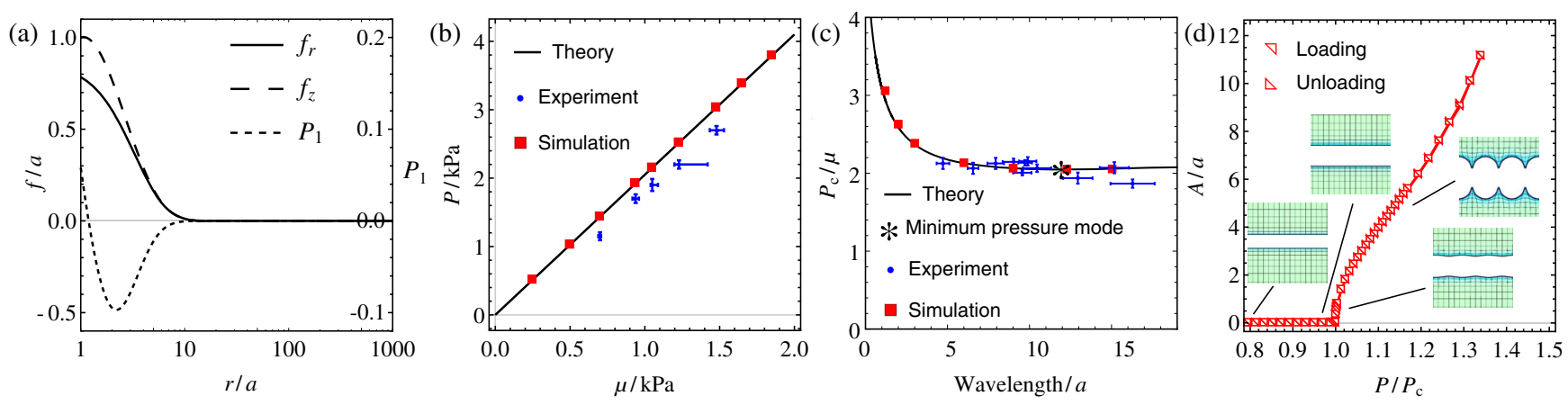

FIG. 3. Theoretical, numerical, and experimental treatment of the peristaltic instability in a channel through a bulk solid. (a) Peristaltic fields for the first unstable mode from the stability analysis, plotted against the undeformed coordinate $r$. (b) Critical pressure for instability as a function of shear modulus. (c) Critical pressure for instability at each wavelength. (d) Simulated amplitude as a function of $P_{\text {in }}$, showing a supercritical transition without hysteresis.

source to vary the pressure in the cavity, and monitored the evolving shape of the channel with a CMOS camera sensor fitted to a Leica stereomicroscope eyepiece. As seen in Fig. 2, at modest pressures the channels simply dilate, in good agreement with the prediction for a channel through a bulk neo-Hookean. However, as seen in Fig. 1 and Video E1 in SM [61], beyond a critical pressure, the channel adopts a new peristaltically undulating morphology. The transition is reversible, and occurs at quasistatic rates of inflation, suggesting a purely elastic mechanical instability.

To understand this transition, we examine the linear stability of uniform dilation to small perturbations, $\mathbf{u}=u_{0} \hat{\mathbf{r}}+\delta \mathbf{u}$, $P=\mu P_{0}+\delta P$, which, in turn, induce first order changes to the deformation gradient $(\delta F=\nabla \delta \mathbf{u})$ and the stress $\left[\delta \sigma=\mu \delta F-\delta P F_{0}^{-T}-\mu P_{0} \delta\left(\operatorname{Det}(F) F^{-T}\right)\right] . \quad$ Expanding Eqs. (2) and (3) to first order, we see the perturbation must satisfy $\nabla \cdot \delta \sigma=0$ (mechanical equilibrium), $\operatorname{Tr}\left(F_{0}^{-1} \cdot \delta F\right)=0$ (incompressibility), and $\left.\left[\delta \sigma+P_{f} \delta\left(\operatorname{Det}(F) F^{-T}\right)\right] \cdot \hat{\mathbf{r}}\right|_{r=a, b}=0$ (boundary conditions). Substituting a peristaltic form,

$$
\begin{gathered}
\delta \mathbf{u}=f_{r}(r) \cos (k z) \hat{\mathbf{r}}+f_{z}(r) \sin (k z) \hat{\mathbf{z}}, \\
\delta P=\mu P_{1}(r) \cos (k z),
\end{gathered}
$$

and using Lagrange's derivative notation, $f^{\prime}=(\partial f / \partial r)$, the condition of incompressibility reduces (see SM [61]) to

$$
R\left(R f_{r}^{\prime}+k r f_{z}\right)+r f_{r}=0,
$$

the equations of mechanical equilibrium become

$$
\begin{aligned}
& k f_{r}\left(r^{2}-R^{2}\right)^{2}+r R^{3}\left(r f_{z}^{\prime \prime}+f_{z}^{\prime}-k^{2} r f_{z}+k r P_{1}\right)=0, \\
& r R^{4}\left(r f_{r}^{\prime \prime}+f_{r}^{\prime}-R P_{1}^{\prime}\right)+r^{2} f_{r}\left[r^{2}-R^{2}\left(k^{2} R^{2}+2\right)\right] \\
& \quad+k R f_{z}\left(r^{2}-R^{2}\right)^{2}=0
\end{aligned}
$$

and the boundary conditions (at both $r=a, b$ ) become

$$
\begin{gathered}
R f_{z}^{\prime}-k r f_{r}=0, \\
r R^{2} f_{r}^{\prime}-r^{2} f_{r}-k r^{2} R f_{z}-R^{3} P_{1}=0 .
\end{gathered}
$$

Given $R \equiv r+u_{0}=\sqrt{a^{2}\left(\lambda^{2}-1\right)+r^{2}}$, Eqs. (10)-(14) form a fourth order generalized eigensystem for the critical degree of dilation $\lambda$ required for instability. Previous authors have solved this system for modestly thick tubes [15] $(b-a \sim a)$ which, like their thin-walled counterparts, first become unstable via long-wavelength modes. Such modes vanish beyond a critical wall thickness, leading these authors to conclude that sufficiently thick pipes are stable. However, inspired by our experimental observations, we use the MATLAB bvp4c routine to search, numerically, for finitewavelength solutions $(k \sim 1 / a)$ in a tube which is sufficiently thick $(b=1000 a)$ to approximate a bulk solid. The first unstable mode occurs at $\lambda=4.824 \ldots$ (requiring $P_{\text {in }}=$ $2.052 \ldots \mu)$ and with finite wavelength $2 \pi / k=12.278 \ldots a$. We plot the form of this solution in Fig. 3(a), showing the fields take maximum values near the cavity, and only penetrate a distance $\sim 10 a$ into the bulk, confirming the outer boundary is effectively at infinity. The variation of threshold pressure with wavelength is plotted in Fig. 3(c). Although the resulting threshold curve is very flat, it does have a minimum (indicated with a star) corresponding to the first unstable mode.

In Figs. 3(b) and 3(c), we also compare these predictions with bespoke $[45,62]$ axisymmetric $[38,63]$ finite element calculations ( $b / a \geq 60$, see details in SM [61]) and threshold measurements obtained in "unloading" experiments, in which a channel is pressurized well beyond the point of instability then quasistatically depressurized until peristalsis vanishes. In particular, we show in Fig. 3(b) that both numerical and experimental cavities are indeed unstable above $P_{\text {in }}=2.05 \ldots \mu$ over a range of gel moduli [64]. This linearity in gel modulus is the hallmark of a purely elastic instability. In finite elements, long cavities select the expected $2 \pi / k=12.278 \ldots a$ wavelength (see Fig. 2 in theory SM [61]), but we can artificially fix the wavelength 

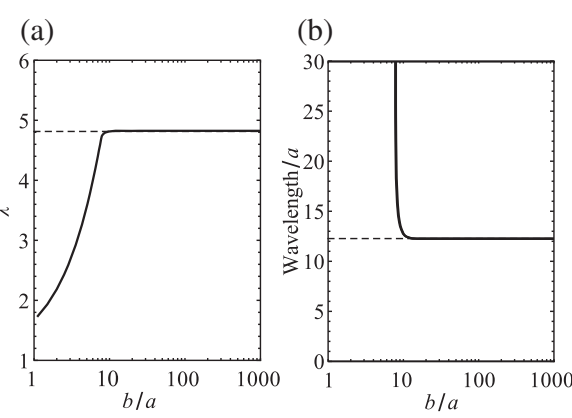

(c)

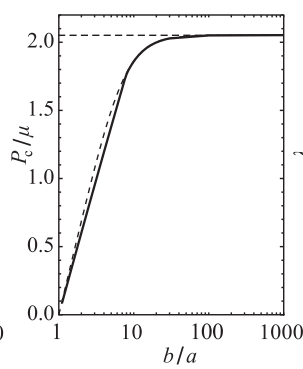

(d)

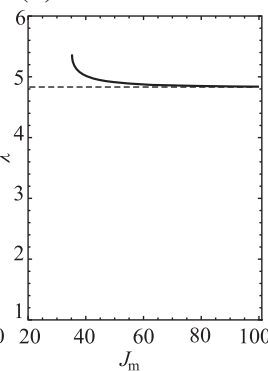

(e)

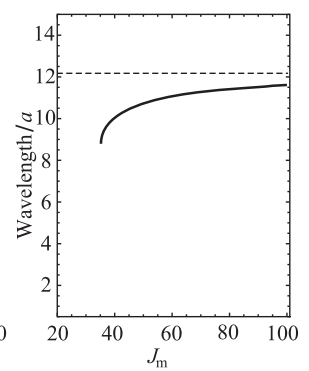

(f)

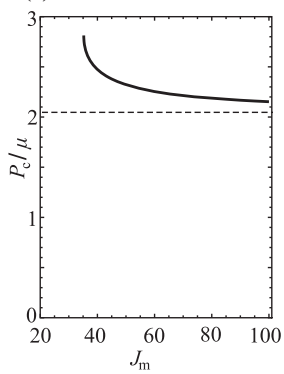

FIG. 4. Effect of finite outer radius (a)-(c) and finite extensibility (d)-(f) on the critical dilation (a),(d), critical wavelength (b),(e), and critical pressure (c),(f) of the peristaltic elastic instability. Straight dashed lines indicate the bulk neo-Hookean solution, while solid lines indicated the full finite $b / a$ solution (a)-(c) and Gent material solution (d)-(f). The curved dashed line in (c) is the pressure, at finite $b / a$, required to produce the bulk critical dilation $\lambda=4.82 \ldots$.

via periodic boundary conditions. In Fig. 3(c), we confirm these alternative wavelengths become unstable at the corresponding (higher) predicted pressures. In experiments, we cannot prescribe a wavelength, but we find the instability naturally occurs with a broad range of wavelengths (a signature of the very flat theoretical wavelengththreshold curve) and, in each case, peristalsis vanishes close to the predicted threshold pressure for that wavelength. The experimental instability is often observed at pressures slightly below the theoretical value, which we attribute to the finite slab thickness.

Unlike stability analysis, our finite element calculations can explore peristalsis beyond threshold. We conducted a numerical loading or unloading cycle in a bulk solid [Fig. 3(d) and Video N1 in SM [61]), which shows the amplitude growing and shrinking continuously above threshold without hysteresis; the instability is supercritical. Repeating this exercise with different wavelengths reveals that the energy-minimizing wavelength lengthens slightly (see Fig. 3 in theory SM [61]) beyond threshold.

Overall, our analysis leads to a simple conclusion: the driver for peristalsis is that, for a given inflated channel volume, the peristaltic form requires less shape change and saves elastic energy. An instructive comparison is to the Plateau-Rayleigh instability $[35,38,65]$, in which peristalsis occurs because it reduces a cylinder's surface area, and hence reduces its surface energy. Here, peristalsis will also reduce the channel's inner surface area, relieving the base-state stretch and saving elastic energy. The base-state stretch Eq. (4) decays into the bulk over the length scale $a$, and the energetically optimal peristaltic fields will penetrate this dilated region, where they relieve strain, but not further where they would add strain and cost energy. As is characteristic of oscillating elastic fields, the peristaltic field's penetration distance is set by their wavelength, resulting in an optimal wavelength proportional to $a$. Since this is a geometric mechanism, we expect peristalsis to be generic in sufficiently deformable elastic channels. In the final section of our study, we investigate how robust peristalsis is to changes in geometry and choice of elastic material.
First, we analyze peristalsis at finite $b / a$, by numerically solving the stability equations [Eqs. (10)-(14)] to plot threshold pressure vs wavelength in cavities with a range of values of $b / a$ (see Fig. 1 in theory SM [61]). The equations only depend on $P_{\text {in }}$ via the dilation $(\lambda)$ it produces, and only depend on $b$ via the outer boundary condition where the fields are in any event decayed. The instability thus has a universal form in all channels with modestly large $b / a$, which all become unstable at the bulk wavelength and dilation. In Figs. 4(a) and 4(b), we show the full form of the threshold dilation, and wavelength as a function of $b / a$. As anticipated, these only deviate from the bulk form when $b / a \lesssim 10$, with wavelength growing and dilation falling in finite systems. However, the critical pressure [Fig. 4(c)] drops from the bulk value rather earlier, as the pressure required to produce this dilation is reduced in finite systems, in accordance with Eq. (6). Peristalsis is replaced by a long-wavelength axisymmetric bulging instability for $b / a \lesssim 7$, the mode previously identified in thick-walled tubes [15], which sets the ultimate limit of peristalsis in finite systems.

Second, we analyze peristalsis beyond Hookean materials. Most elastomers or gels are neo-Hookean at modest strains, but have a finite extensibility, owing to their finite length chains. The resulting stain stiffening is captured by the Gent model [66], $W(F)=$ $-\frac{1}{2} \mu J_{m} \log \left\{1-\left[\operatorname{Tr}\left(F . F^{T}\right)-3\right] / J_{m}\right\}-P[\operatorname{Det}(F)-1]$, which reproduces the neo-Hookean energy when the strain measure $\operatorname{Tr}\left(F \cdot F^{T}\right)-3$ is small, but diverges when it approaches $J_{m}$, a phenomenological parameter encoding finite extensibility. Repeating the bulk linear-stability analysis, we find the base state is identical, while the stability equations become more complicated, but still admit numerical solution. We display the critical dilation, wavelength, and pressure as a function of $J_{m}$ in Figs. 4(d)-(f), showing that finite extensibility generates only modest changes in the form of peristalsis, but eliminates the instability entirely for $J_{m}<35$, corresponding to materials with limiting uniaxial extension factor of $\lambda \lesssim 6$. 

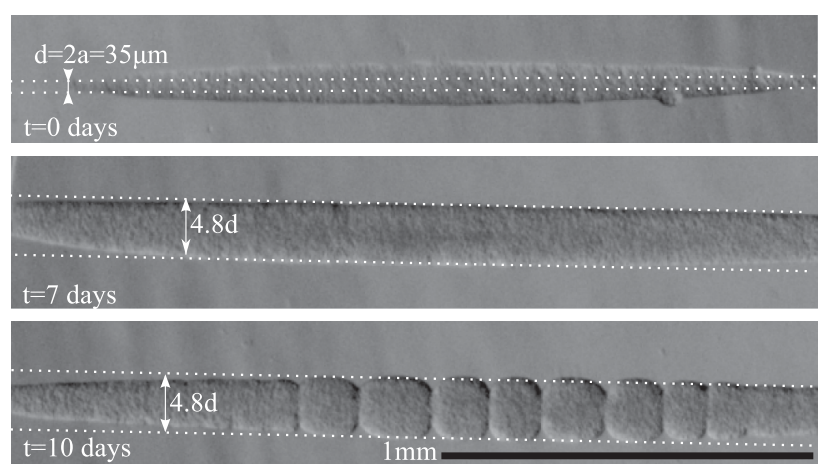

FIG. 5. Instability when an embryonic stem cell tissue grows inside a channel. Dashed lines indicate initial channel diameter $d=2 a$ (top) and predicted threshold diameter for instability (4.8d, middle, bottom).

In summary, we have shown that a cylindrical channel through a soft solid will spontaneously adopt a peristaltically undulating shape when bearing a pressure comparable to the solid's shear modulus. The instability takes a simple form in channels through bulk neo-Hookean solids, occurring at a critical pressure $(2.05 \ldots \mu)$ proportional to the channel shear modulus, and with a wavelength which is a multiple of the cavity radius. Assuming a finite threshold and wavelength, these scalings are an inevitable consequence of the scale invariance of elasticity, which means $a$ is the only length scale in the problem, and $\mu$ is the only stress scale. Several one-length-scale elastic fingering phenomena produce analogous wavelengths [30-32,67-70], and solid cavitation of bulk spherical cavities follows the same stress scaling $\left(P_{c}=2.5 \mu\right)$, though bulk peristalsis of cylindrical cavities requires less pressure.

Peristalsis is also expected in finite solids, provided $b / a \gtrsim 7$, and strain-stiffening materials provided they are sufficiently deformable. In some less-deformable elastic media, the strain concentrations associated with peristalsis will precipitate fracture and failure, suggesting peristalsis places a fundamental limit on the pressure a channel can bear, just as Euler-buckling limits loading of columns. Conversely, in sufficiently deformable solids, peristalsis offers a route to reversibly introduce periodicity into a channel, potentially allowing a highly reflective photonic or phononic stop band [71,72] to be switched within a deformable waveguide.

We speculate that the peristaltic instability will also manifest in biology, sculpting channels during both pathological and developmental processes. Indeed, we first observed peristalsis in hydrogel channels containing growing mouse embryonic stem cell tissues (Fig. 5). More precisely, we injected a dense suspension of murine embryonic stem cells into a 35- $\mu$ m-diameter $(a=17.5 \mu \mathrm{m})$ channel, which rapidly adhered into a monolithic tissue. This tissue grew for 10 days within the channel, nourished by cell culture media (see Refs. [73,74] and SM [61]). Such embryonic stem cell tissue is a soft incompressible solid with a finite yield stress, and dilates the channel as it grows. The tissue solidity arises because the cells are both individually elastic (with moduli $\sim 100 \mathrm{~Pa}$ [75]) owing to their cytoskeleton, and adhere to each other by expression of E-cadherin [76]: we found disrupting either aspect (using Cytochalasin D [77] or EDTA [78], respectively; see Fig. 3 in experimental SM [61]) led to a tissue which could no longer deform the hydrogel. With the undisrupted culture, peristalsis appeared on day seven, when the central channel dilation achieved the expected threshold of $\lambda \approx 4.8$. As in pressure-controlled experiments, peristalsis had uneven wavelengths, varying between $7 a$ and $12 a$, reflecting the instability's flat dispersion curve.

In biology, one commonly encounters thin-walled tubes through soft tissues. Such tubes can be included in the current framework by adding a thin tube of different elastic material, with modulus $\mu_{\text {tube }}=\eta \mu$, at the inner radius of the channel. Under inflation this system must span from conventional long-wavelength tube instabilities at high $\eta$ to finite-wavelength peristalsis at low $\eta$, and mapping this transition is likely to be a rich and biologically relevant subject for future investigations.

We thank T. Tallinen, on whose code our finite element calculations are based. N.C. thanks the Royal Thai Government Scholarship for funding. K. L. received support from the Biotechnology and Biological Sciences Research Council (BB/ J014427/1) and Lithuanian Science Council Postdoctoral Award (code 09.3.3- LMTK-712-02-0067). S.S. is funded by a Wellcome Senior Investigator Award (103788/Z/14/Z) and Wellcome Technology award (108438/Z/15/Z).

[1] T. Pedley, Blood flow in arteries and veins, in Perspectives in Fluid Dynamics, edited by G. Batchelor, H. Moffat, and M. Worster (Cambridge University Press, Cambridge, 2000), p. 105.

[2] M. A. Unger, H.-P. Chou, T. Thorsen, A. Scherer, and S. R. Quake, Science 288, 113 (2000).

[3] Y. Xia and G. M. Whitesides, Annu. Rev. Mater. Sci. 28, 153 (1998).

[4] J. C. McDonald, D. C. Duffy, J. R. Anderson, D. T. Chiu, H. Wu, O. J. Schueller, and G. M. Whitesides, Electrophoresis 21, 27 (2000).

[5] T. Thorsen, S. J. Maerkl, and S. R. Quake, Science 298, 580 (2002).

[6] H.-P. Chou, M. A. Unger, and S. R. Quake, Biomed. Microdevices 3, 323 (2001).

[7] I. E. Araci, B. Su, S. R. Quake, and Y. Mandel, Nat. Med. 20, 1074 (2014).

[8] A. Koh et al., Sci. Transl. Med. 8, 366ra165 (2016).

[9] A. Mallock, Proc. R. Soc. London 49, 458 (1891).

[10] A. H. Corneliussen and R. Shield, Arch. Ration. Mech. Anal. 7, 273 (1961).

[11] H. Alexander, Int. J. Mech. Sci. 13, 87 (1971). 
[12] R. T. Shield, ZAMP 23, 16 (1972).

[13] R. W. Ogden, Proc. R. Soc. A 326, 565 (1972).

[14] D. Haughton and R. Ogden, J. Mech. Phys. Solids 27, 179 (1979).

[15] D. Haughton and R. Ogden, J. Mech. Phys. Solids 27, 489 (1979).

[16] A. Gent, Rubber Chem. Technol. 72, 263 (1999).

[17] Y. Fu, S. Pearce, and K.-K. Liu, Int. J. Nonlinear Mech. 43, 697 (2008).

[18] F. Meng, J. Z. Y. Chen, M. Doi, and Z. Ouyang, AIChE J. 60, 1393 (2014).

[19] L. Euler, Methodus inveniendi lineas curvas maximi minimive proprietate gaudentes sive solutio problematis isoperimetrici latissimo sensu accepti, Lausannae et Genevae (1744), p. 231, http://eulerarchive.maa.org/docs/ originals/E065a.Additamentum I: De curvis elasticis, http:// eulerarchive.maa.org/docs/originals/E065g.

[20] M. Biot, Mechanics of Incremental Deformations: Theory of Elasticity and Viscoelasticity of Initially Stressed Solids and Fluids, Including Thermodynamic Foundations and Applications to Finite Strain (Wiley, New York, 1965).

[21] V. Trujillo, J. Kim, and R. C. Hayward, Soft Matter 4, 564 (2008).

[22] S. Cai, D. Chen, Z. Suo, and R. C. Hayward, Soft Matter 8, 1301 (2012).

[23] E. Hohlfeld and L. Mahadevan, Phys. Rev. Lett. 106, 105702 (2011).

[24] J. Dervaux and M. B. Amar, Annu. Rev. Condens. Matter Phys. 3, 311 (2012).

[25] T. Tallinen, J. S. Biggins, and L. Mahadevan, Phys. Rev. Lett. 110, 024302 (2013).

[26] H. G. Allen, Analysis and Design of Structural Sandwich Panels: The Commonwealth and International Library: Structures and Solid Body Mechanics Division (Pergamon, New York, 1969).

[27] E. Cerda and L. Mahadevan, Phys. Rev. Lett. 90, 074302 (2003).

[28] K. R. Shull, C. M. Flanigan, and A. J. Crosby, Phys. Rev. Lett. 84, 3057 (2000).

[29] B. Saintyves, O. Dauchot, and E. Bouchaud, Phys. Rev. Lett. 111, 047801 (2013).

[30] J. S. Biggins, B. Saintyves, Z. Wei, E. Bouchaud, and L. Mahadevan, Proc. Natl. Acad. Sci. U.S.A. 110, 12545 (2013).

[31] S. Mora, T. Phou, J.-M. Fromental, and Y. Pomeau, Phys. Rev. Lett. 113, 178301 (2014).

[32] J. S. Biggins, Z. Wei, and L. Mahadevan, Europhys. Lett. 110, 34001 (2015).

[33] S. Lin, T. Cohen, T. Zhang, H. Yuk, R. Abeyaratne, and X. Zhao, Soft Matter 12, 8899 (2016).

[34] S. Lin, Y. Mao, R. Radovitzky, and X. Zhao, J. Mech. Phys. Solids 106, 229 (2017).

[35] S. Mora, T. Phou, J.-M. Fromental, L. M. Pismen, and Y. Pomeau, Phys. Rev. Lett. 105, 214301 (2010).

[36] M. Taffetani and P. Ciarletta, Phys. Rev. E 91, 032413 (2015).

[37] P. Ciarletta and M. B. Amar, Soft Matter 8, 1760 (2012).

[38] C. Xuan and J. Biggins, Phys. Rev. E 95, 053106 (2017).

[39] H. Alexander, Int. J. Eng. Sci. 9, 151 (1971).
[40] A. Gent, Int. J. Nonlinear Mech. 40, 165 (2005).

[41] A. Gent and P. Lindley, Proc. R. Soc. A 249, 195 (1959).

[42] J. M. Ball, Phil. Trans. R. Soc. A 306, 557 (1982).

[43] A. N. Gent, Rubber Chem. Technol. 63, 49 (1990).

[44] A. E. Hirst, Jr., V. J. Johns, and S. W. Kime, Jr., Medicine 37, 217 (1958).

[45] T. Tallinen, J. Y. Chung, J. S. Biggins, and L. Mahadevan, Proc. Natl. Acad. Sci. U.S.A. 111, 12667 (2014).

[46] T. Tallinen, J. Y. Chung, F. Rosseau, N. Girard, J. Lefevre, and L. Mahadevan, Nat. Phys. 12, 588 (2016).

[47] T. Savin, N. A. Kurpios, A. E. Shyer, P. Florescu, H. Liang, L. Mahadevan, and C. J. Tabin, Nature (London) 476, 57 (2011).

[48] A. E. Shyer, T. Tallinen, N. L. Nerurkar, Z. Wei, E. S. Gil, D. L. Kaplan, C. J. Tabin, and L. Mahadevan, Science 342, 212 (2013).

[49] J. Dervaux, Y. Couder, M.-A. Guedeau-Boudeville, and M. B. Amar, Phys. Rev. Lett. 107, 018103 (2011).

[50] P. Ciarletta and M. B. Amar, Int. J. Nonlinear Mech. 47, 248 (2012).

[51] M. Kücken and A. Newell, Europhys. Lett. 68, 141 (2004).

[52] M. Diab, T. Zhang, R. Zhao, H. Gao, and K.-S. Kim, Proc. R. Soc. A 469, 20120753 (2013).

[53] M. J. Razavi, R. Pidaparti, and X. Wang, Phys. Rev. E 94, 022405 (2016).

[54] J. Shim, C. Perdigou, E. R. Chen, K. Bertoldi, and P. M. Reis, Proc. Natl. Acad. Sci. U.S.A. 109, 5978 (2012).

[55] D. Yang, B. Mosadegh, A. Ainla, B. Lee, F. Khashai, Z. Suo, K. Bertoldi, and G. M. Whitesides, Adv. Mater. 27, 6323 (2015).

[56] J. Marthelot, P.-T. Brun, F. L. Jiménez, and P. M. Reis, Phys. Rev. Mater. 1, 025601 (2017).

[57] D. Wang, N. Cheewaruangroj, Y. Li, G. McHale, Y. Jiang, D. Wood, J. S. Biggins, and B. B. Xu, Adv. Funct. Mater. 28, 1704228 (2018).

[58] R. S. Rivlin, Phil. Trans. R. Soc. A 240, 459 (1948).

[59] H. M. James and E. Guth, J. Chem. Phys. 11, 455 (1943).

[60] J. Tang, J. Li, J. J. Vlassak, and Z. Suo, Extreme Mech. Lett. 10, 24 (2017).

[61] See Supplemental Material at http://link.aps.org/ supplemental/10.1103/PhysRevLett.122.068003 for videos and further experimental, theoretical and numerical details.

[62] T. Tallinen and J. S. Biggins, Phys. Rev. E 92, 022720 (2015).

[63] C. Xuan and J. Biggins, Phys. Rev. E 94, 023107 (2016).

[64] J. R. Tse and A. J. Engler, Curr. Protoc. Cell Biol. 47, 10.16.1 (2010).

[65] Lord Rayleigh, Proc. London Math. Soc. s1-10, 4 (1878).

[66] A. Gent, Rubber Chem. Technol. 69, 59 (1996).

[67] A. Ghatak and M. K. Chaudhury, Langmuir 19, 2621 (2003).

[68] M. Adda-Bedia and L. Mahadevan, Proc. R. Soc. A 462, 3233 (2006).

[69] M. K. Chaudhury, A. Chakrabarti, and A. Ghatak, Eur. Phys. J. E 38, 82 (2015).

[70] J. S. Biggins and L. Mahadevan, Soft Matter 14, 7680 (2018).

[71] Lord Rayleigh, Phil. Mag. J. Sci. 26, 256 (1888). 
[72] K. Bertoldi and M. C. Boyce, Phys. Rev. B 77, 052105 (2008).

[73] J. M. Rhee, M. K. Pirity, C.S. Lackan, J. Z. Long, G. Kondoh, J. Takeda, and A.-K. Hadjantonakis, Genesis 44, 202 (2006).

[74] X. Wang and P. Yang, J. Visualized Exp. 17, 825 (2008).
[75] S. Pagliara, K. Franze, C. R. McClain, G. W. Wylde, C. L. Fisher, R. J. Franklin, A. J. Kabla, U. F. Keyser, and K. J. Chalut, Nat. Mater. 13, 638 (2014).

[76] T. Pieters and F. Van Roy, J. Cell Sci. 127, 2603 (2014).

[77] J. F. Casella, M. D. Flanagan, and S. Lin, Nature (London) 293, 302 (1981).

[78] T. L. Le, J. L. Stow et al., J. Cell Biol. 146, 219 (1999). 